\title{
Smart Optimization of Proactive Control of Petroleum Reservoir
}

Edyta Kukl, Michał Kuk, Damian Janiga, Paweł Wojnarowski, Jerzy Stopa AGH University of Science and Technology, Poland

\section{Abstract}

Artificial Intelligence plays an increasingly important role in many industrial applications as it has great potential for solving complex engineering problems. One of such applications is the optimization of petroleum reservoirs production. It is crucial to produce hydrocarbons efficiently as their geological resources are limited. From an economic point of view, optimization of hydrocarbon well control is an important factor as it affects the whole market. The solution proposed in this paper is based on state-of-the-art artificial intelligence methods, optimal control, and decision tree theory. The proposed idea is to apply a novel temporal clustering algorithm utilizing an autoencoder for temporal dimensionality reduction and a temporal clustering layer for cluster assignment, to cluster wells into groups depending on the production situation that occurs in the vicinity of the well, which allows reacting proactively. Then the optimal control of wells belonging to specific groups is determined using an auto-adaptive decision tree whose parameters are optimized using a novel sequential model-based algorithm configuration method. Optimization of petroleum reservoirs production translates directly into several economic benefits: reduction in operation costs, increase in the production effectiveness and increase in overall income without any extra expenditure as only control is changed.

Keywords: hydrocarbon wells production; control optimization; artificial intelligence; temporal clustering; auto-adaptive decision tree

JEL classification: $\mathrm{O} 31$

Acknowledgments: The paper was performed within the frame of AGH-UST statutory research grant No. 16.16.190.779 Faculty of Drilling, Oil, and Gas, Department of Petroleum Engineering.

Paper type: Research article

Received: Mar 13, 2021

Accepted: Jun 24, 2021

DOI: $10.54820 /$ ASQF9458

\footnotetext{
${ }^{1}$ Edyta Kuk is currently with Hitachi ABB Power Grids Research, ul. Pawia 7, 31-154

Kraków, Poland.
} 


\section{Introduction}

Artificial Intelligence plays an increasingly important role in many industrial applications. It constitutes a branch of computer science focusing on machines having some human-specific characteristics e.g., learning from gained experience and use of this knowledge in new situations (Burggräf et al., 2018). Artificial intelligence methods allow for assistance or replacement of humans in solving non-trivial problems that could potentially be solved only by humans (Shteimberg et al., 2012). Moreover, artificial intelligence has great potential for solving complex engineering problems. As a result, artificial intelligence allows for the automatization of operations, reducing the amount of human labor.

As applications of artificial intelligence methods are constantly growing, more and more methods are being developed. In recent years, one of the most developing artificial intelligence areas is deep learning. This subgroup of machine learning includes structures e.g., deep neural networks (DNN), recurrent neural networks (RNN), convolutional neural networks (CNN), deep belief networks (DBN), as well as graph neural networks (GNN) (Pang et al., 2020). Thanks to their performance deep learning can be applied to high-dimensional data as well as temporal data (Pang et al., 2020). One of the most widely applied deep neural networks is an auto-encoder, which allows for learning efficient data codings in an unsupervised manner (Kramer, 1991). The autoencoder is responsible for dimensionality reduction and reconstruction of the original signal based on the data with a reduced dimensionality (Madiraju et al., 2018). The typical objective function is the mean square error computed between the original signal and the recreated after dimensionality reduction.

Artificial intelligence methods (including machine learning methods) are used to a wide range of problems in the petroleum industry, such as the interpretation of well logging, optimization of the drilling process as well as subsequent production (Mikołajczak et al., 2018; Czarnota et al. 2018). One of such applications is the optimization of petroleum reservoirs production (Janiga et al., 2017). It is crucial to produce hydrocarbons efficiently as their geological resources are limited (Kuk et al., 2020). From an economic point of view, optimization of hydrocarbon wells control is an important factor as it affects the whole market.

This paper presents a novel approach to petroleum reservoirs control which is focused on the proactive control approach and its intelligent optimization. The idea of this paper is to combine the optimal control theory with decision tree theory and enrich them using machine learning algorithms, such as a deep temporal clustering neural network and sequential model-based algorithm configuration method. In the second part of this paper, the overview of the algorithms used in the proposed approach is contained. In the third section, a detailed description of the developed approach is included. The fourth part is focused on the discussion about the strengths and weaknesses of the proposed solution. While the last section includes the conclusions.

\section{Methodology}

The approach proposed in this paper for the smart optimization of the proactive control of the petroleum reservoirs is based on the optimal control theory, an innovative concept of auto-adaptive decision trees as well as a novel temporal clustering algorithm, which constitutes a state-of-the-art artificial intelligence method. 


\section{Optimal control}

The optimal control of petroleum reservoirs aims to determine such a sequence of actions defining a production strategy that extremizes (maximizes or minimizes) the assumed objective function, considering the practical limitations (Ramirez, 1987). Control optimization can focus on minimization of water production which reduces cost, maximization of gas/oil production which increases income, or directly maximization of the economic value of the whole project.

The problem to be solved in this paper is to determine such petroleum reservoir control that extremizes (maximize or minimize) an assumed objective function. Due to the complicated characteristics of hydrocarbon reservoirs, the possibility of application of the classical optimal control determination methods is limited. Hence, the approach developed in this paper utilizes machine learning methods that can efficiently solve complex problems.

\section{Decision Trees}

One of the possibilities to determine control actions that should be taken in a particular situation is to present the decision process as a decision tree. It is a structure in which attributes are assigned to tree internal nodes, their limit values are assigned to tree branches, and the control actions that should be taken are stored in the leaves of the tree (Shaowen et al., 2015). Such representation of a decision process allows its unambiguous interpretation. The path from the root (top of a decision tree) to a specific leaf represents a conjunction of conditions that determine the decision to be taken.

As the proper selection of the limit values for tree attributes is a challenge, the concept of the auto-adaptive decision trees was proposed (Kuk, 2019). This concept utilizes a decision tree, in which limit values are parametrized to optimize the whole decision process. In this approach, final actions can also be parametrized as presented in Figure 1. These parameters are then optimized with an advanced machine learning method. The sequential model-based algorithm configuration method is applied in this approach. While optimization is in progress, this method learns which values of parameters can potentially generate the best results (Hutter et al., 2013). Such an approach allows to fully automatically determine the optimal process control.

In the proposed application, attributes contained in tree nodes can represent petroleum reservoir characteristics, such as a reservoir pressure or they can be related to the production process, e.g., well flow rate. These attributes are determined with the use of a reservoir numerical simulator which is also utilized during the tree parameters optimization to calculate the objective function value. 
Figure 1

Parametrized Decision Tree

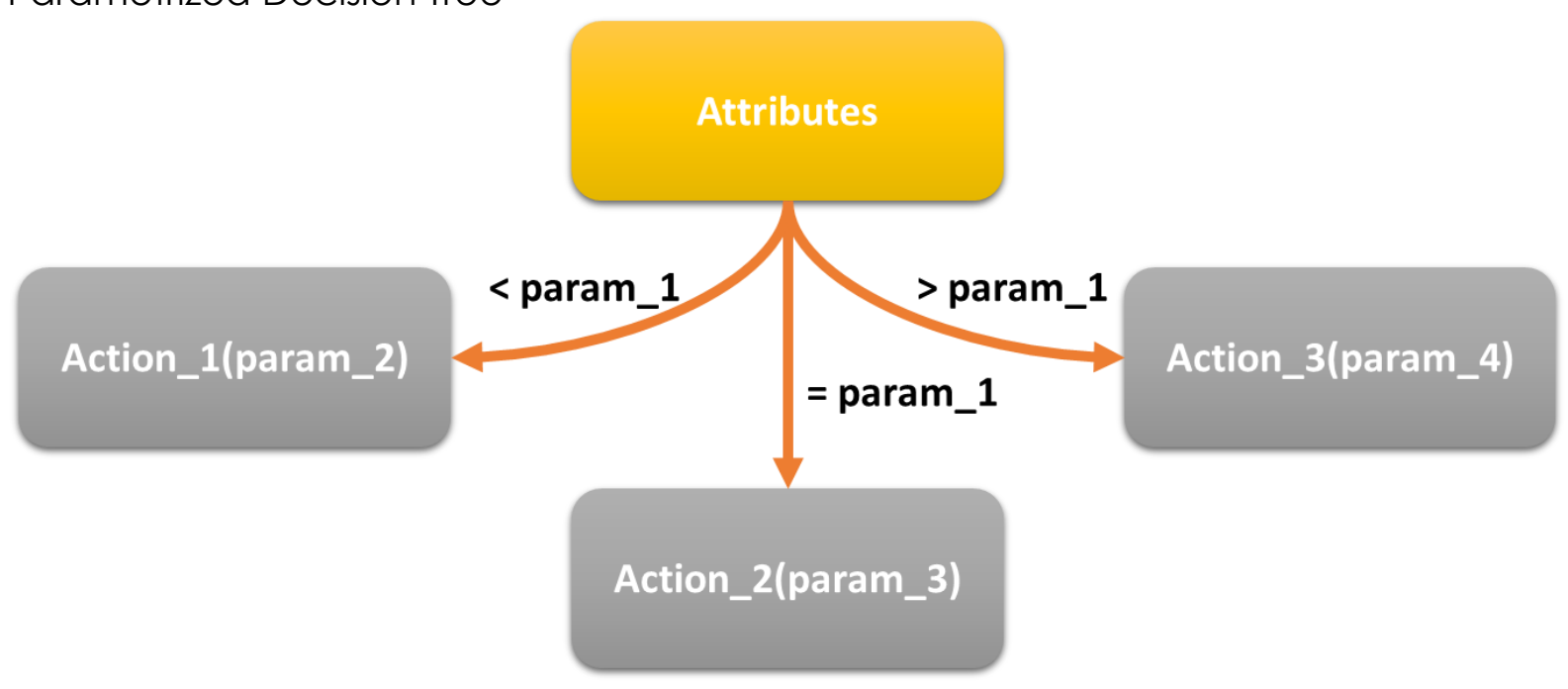

Source: Author's illustration

\section{Temporal Clustering}

Temporal clustering constitutes an unsupervised machine learning method that allows to group time series data based on their similarity into meaningful clusters (Madiraju et al., 2018). The main idea of the temporal clustering algorithm applied in this paper is the transformation of the time series data into a low dimensional latent space with the use of a deep neural network (Madiraju et al., 2018). To uncover the latent dimensions along which the temporal unlabeled data split into classes, an autoencoder is applied in this algorithm for temporal dimensionality reduction, and a temporal clustering layer is utilized for cluster assignment. The idea of the autoencoder is to reduce data dimensionality into latent representations of these data and then reconstruct the original data based on the latent space. Whereas a clustering layer performs non-parametric clustering of latent representation of data and finally finds temporal dimensions along which the data split into groups.

In our problem, the temporal clustering algorithm application aims to cluster wells located in a particular petroleum reservoir into groups depending on the production situation that occurs in the vicinity of each well. The input data into the temporal clustering algorithm include:

- reservoir parameters e.g., reservoir pressure or water/gas saturation in the vicinity of a well,

- production wells data, such as well pressure or flow rate.

Based on this information the algorithm returns the production stage at which a given well is in a particular moment, as presented in Figure 2. Such an approach allows one to react proactively and take control of actions before an undesired situation occurs. As the operation of wells located on petroleum reservoirs is mainly based only on a trial-and-error basis (reactive control approach), such a proactive approach allows enhancing the economic efficiency of the production process (Abreu, 2015). 
Figure 2

Temporal Clustering Algorithm Application to Well Control

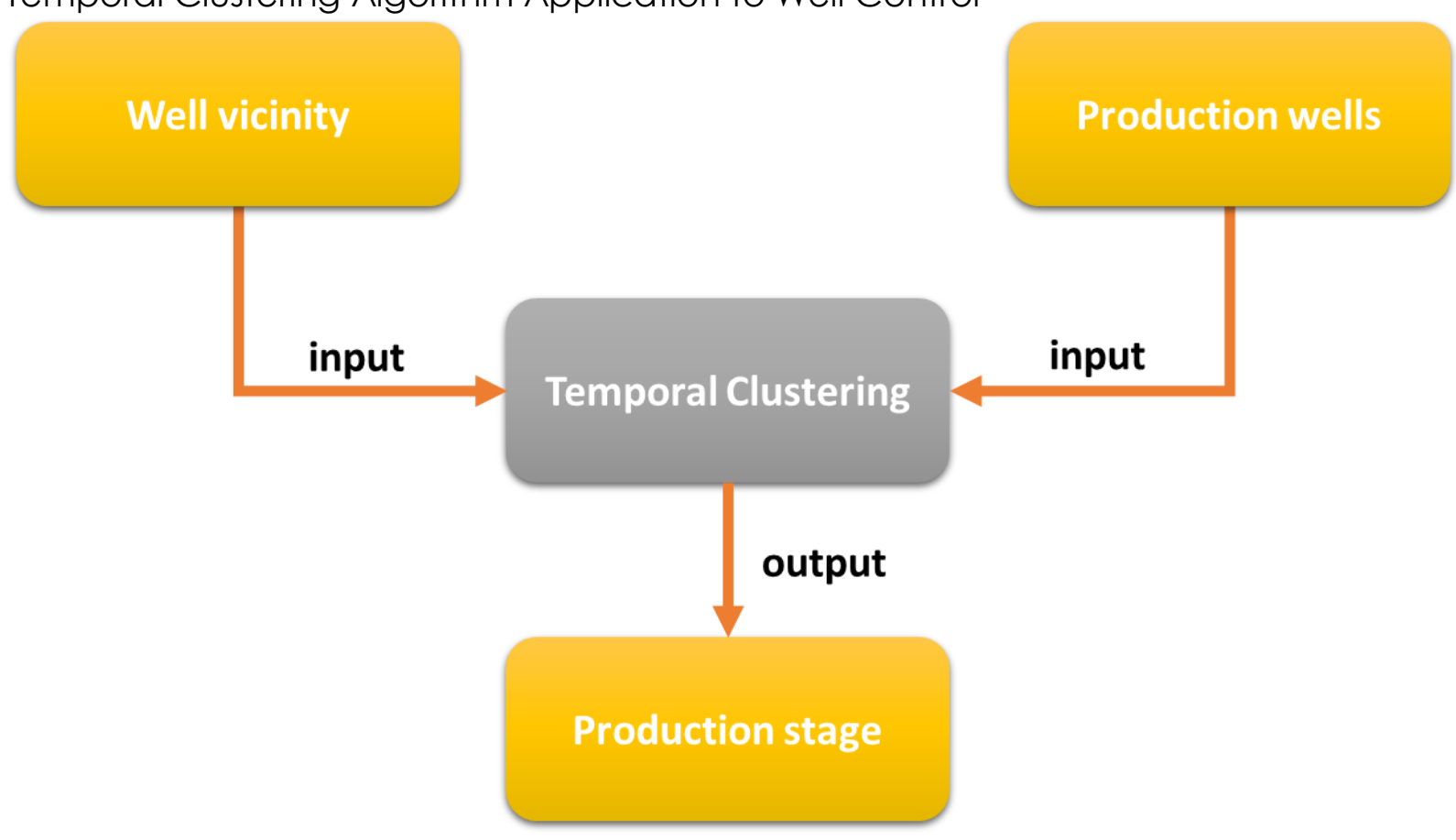

Source: Author's illustration

\section{Results}

Our solution to the petroleum reservoirs' optimal control problem is based on the proactive control approach combined with its smart optimization. The proposed idea is to apply a deep temporal clustering algorithm to cluster wells into groups depending on the production situation that occurs in the vicinity of the well, which allows reacting proactively. Then the optimal control of wells belonging to specific groups is determined using an auto-adaptive decision tree whose parameters are optimized using a machine learning-based optimization method.

In the proposed decision model, presented in Figure 3, reservoir parameters from the vicinity of a production well and the ground production data are determined with the use of a reservoir numerical simulator of a hydrocarbon reservoir. Then, these attributes are combined and used as the input data to the deep temporal clustering algorithm. Based on the analyzes of these data algorithm automatically determines the current production stage of the well. Then, the auto-adaptive decision tree is applied to determine the control actions to be performed within a specific time step depending on the determined production stage of the well. These actions are parameterized and optimized with the sequential model-based algorithm configuration method. Such formulation of the decision process enables automatic determination of the intelligent proactive control of hydrocarbon wells and allows its unambiguous physical interpretation. 
Figure 3

Proposed Decision Model

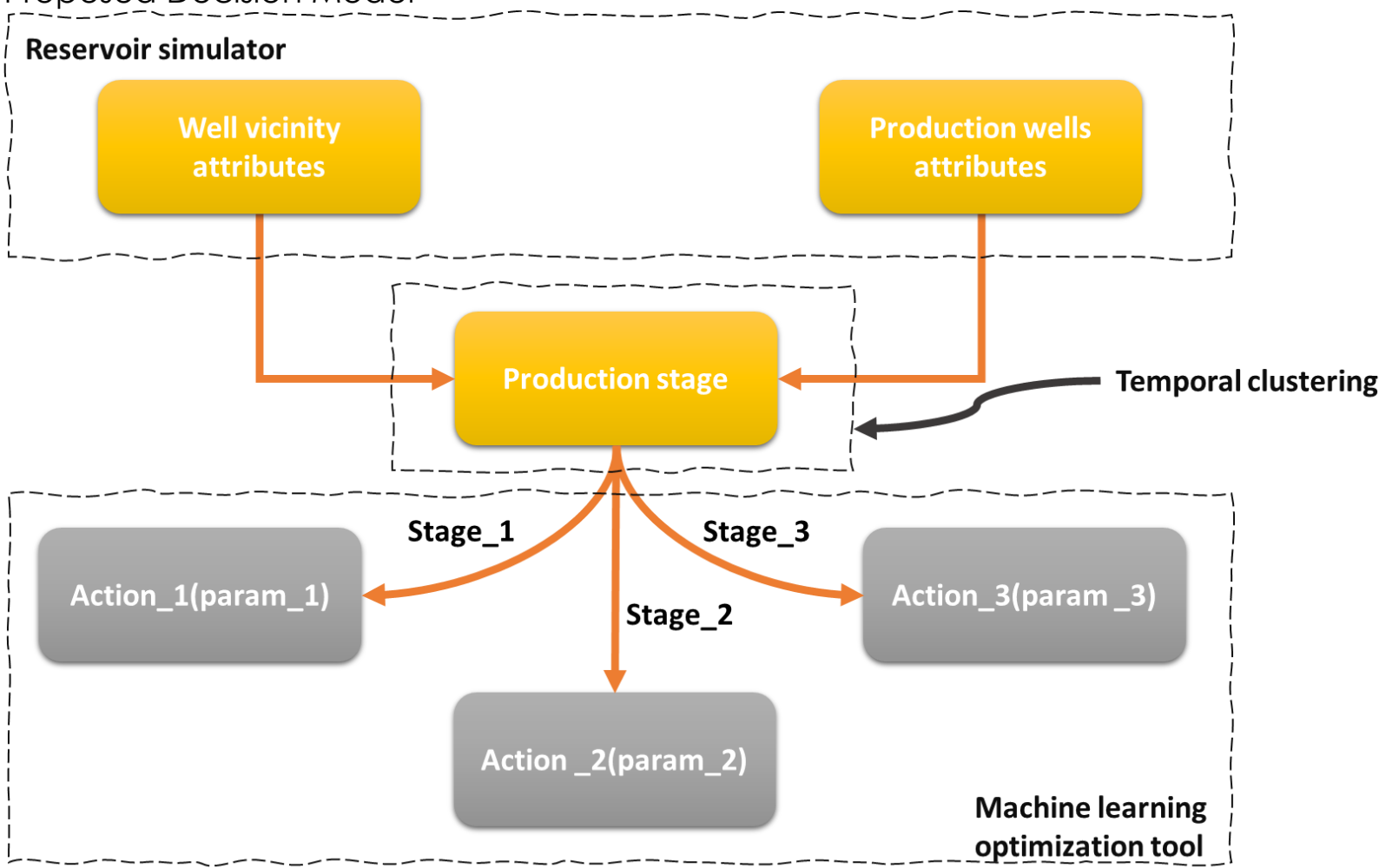

Source: Author's illustration

To apply the proposed decision model, it was also needed to create the computational procedure that would allow all the methods used to be combined. This algorithm is also proposed in this paper.

To apply the deep temporal clustering method, it is firstly needed to learn this algorithm based on the historical data on how properly detect the production stage of a well. As a result, the trained algorithm can predict the production stage of a well based on new data.

Then in the proposed algorithm, for a given set of parameters defining the control actions, in each time step:

- reservoir simulator returns values of reservoir parameters in the area surrounding the analyzed well and its production data,

- based on these attributes' values, the deep temporal clustering algorithm predicts the current production stage of a given well,

- the determined production stage is transferred to the reservoir simulator,

- the proposed decision scheme is also directly implemented in the reservoir simulator,

- the proposed decision scheme determines the well control prevailing in the next time step dependent on the current production stage of the well.

Such a process is repeated for the entire duration of the considered production process simulation. Then, based on the final results of the reservoir simulation the objective function value is calculated. It is used as an input to the machine learning-based optimization tool. The sequential model-based algorithm configuration method used, outputs the next set of parameters that can bring more favorable results. These paraments are fed into a new reservoir simulation 
run and the whole procedure started one more time. After a given number of the whole procedure iterations, the values of the optimized parameters are determined and as a result, the optimal control of the well is obtained.

The block diagram of the whole proposed system is shown in Figure 4.

Figure 4

Proposed System for the Intelligent Proactive Control of Hydrocarbon Wells

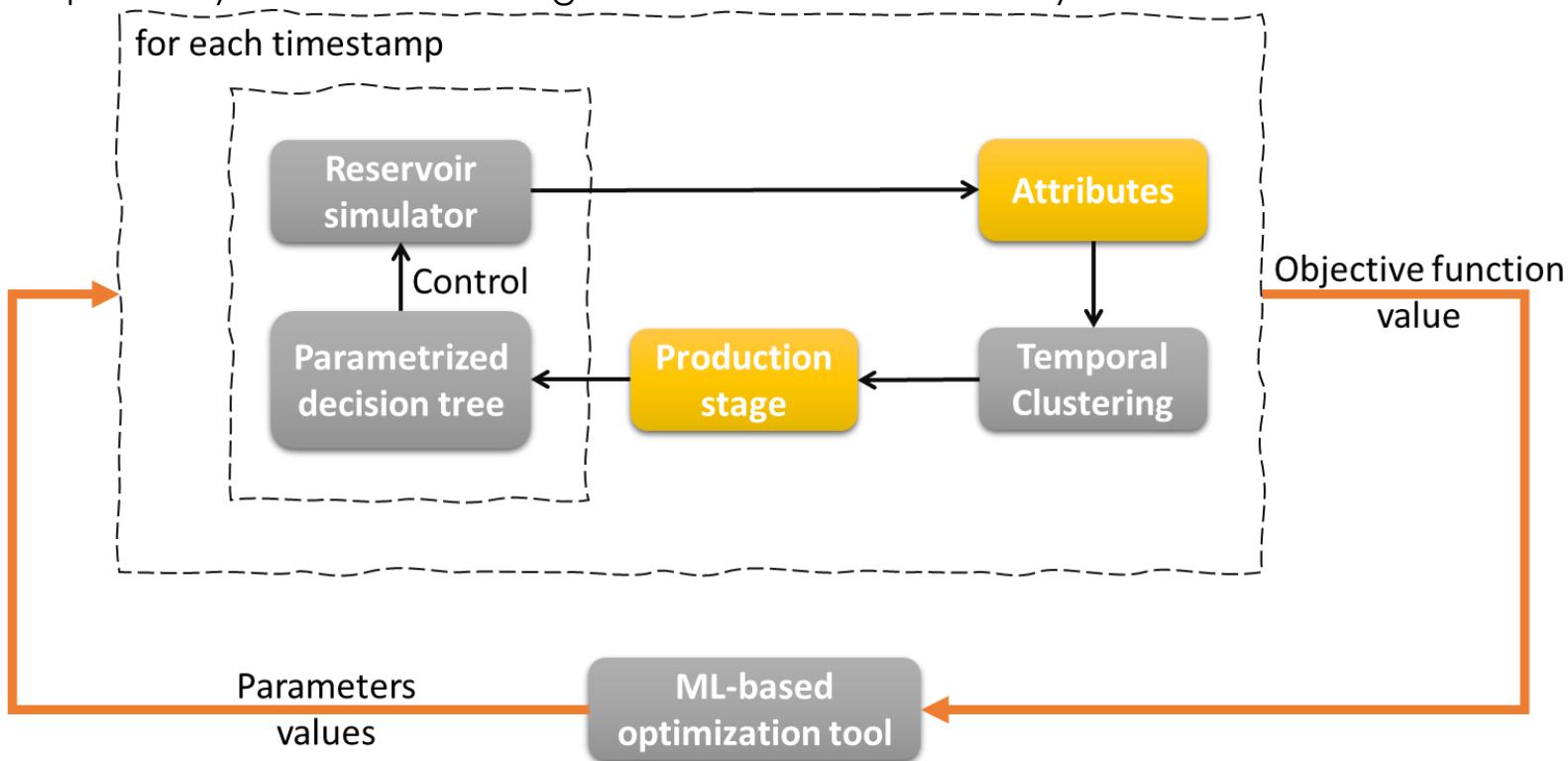

Source: Author's illustration

The software linking the reservoir simulator and the machine learning type optimization tool is implemented in the Python programming language. A combination of the reservoir simulator and the deep temporal clustering algorithm is also implemented in the Python programming language. Such implementation of the proposed algorithm enables full automation of the intelligent proactive well control determination.

\section{Discussion}

The approach proposed in this paper enables better management of petroleum reservoirs. Thanks to the use of advanced machine learning algorithms, the optimal control of a well can be determined also in situations that are not trivial for a human. In the developed solution, the final structure of the proposed general form of the decision scheme is developed by domain experts based on the best industrial practices. It makes the decision process understandable for the operator, which is especially important considering real applications. In addition, the decision scheme can be adapted to any particular production process which makes the proposed method general.

As reservoir parameters in the area surrounding the well are also taken into consideration in the created decision process model, it allows reacting proactively. However, the limitation of the proposed proactive wells control is that there is a need to use a reservoir simulator which may be a difficulty in the case of real-time applications.

As optimization of petroleum reservoirs production may ultimately generate several economic benefits, the implementation of the presented results can:

- Reduce operation costs, 
- Increase the production effectiveness,

- Increase the total income without increasing expenses because only the well control is changed.

All these advantages of the proposed solution can ultimately increase the profit of the petroleum company what is important as it affects the whole market. Moreover, from the environmental point of view, the intelligent proactive control of hydrocarbon wells enables:

- Better utilization of limited hydrocarbon geological resources thanks to increased effectiveness of the production process,

- Reduction of the negative impact of the production process on the natural environment which can translate into a positive perception of ecological environments.

\section{Conclusion}

In this paper, the novel approach for the proactive control of petroleum reservoir combined with its smart optimization was proposed. From an economic point of view, optimization of hydrocarbon wells control is an important factor as it affects the whole market.

The main idea of the approach proposed in this paper is to analyze reservoir parameters in the vicinity of a hydrocarbon well with the use of advanced artificial intelligence-based algorithms to automatically determine the intelligent well proactive control. In the proposed solution wells are clustered into groups with a deep neural network depending on the production situation that occurs in the vicinity of the well, which allows reacting proactively. Then the optimal control of wells belonging to specific groups is determined using a machine learning type optimization method.

The novel approach to the optimization of the hydrocarbon wells control that is proposed in this paper is based on advanced artificial intelligence methods, optimal control theory, and decision tree theory. The approach developed in the study reported in this paper enables better management of petroleum reservoirs, also in situations that are not trivial for a human. However, the developed solution is still understandable for the operator. Thanks to the analysis of reservoir parameters in the vicinity of the well it allows to react proactively but requires utilization of a reservoir simulator. Furthermore, the obtained results implementation may ultimately generate several economic and environmental benefits.

Future work of the study reported here could include an application of the developed solution to the optimization of the real petroleum reservoir production processes and validation of the obtained results.

\section{References}

1. Abreu, A. C. A., Booth, R., Bertolini, A., Prange, M., Bailey, W. J., Teixeira, G., Emetick, A., Pacheco M. A. (2015), "Proactive and Reactive Strategies for Optimal Operational Design: An Application in Smart Wells", OTC Brasil, Rio de Janeiro, Brazil, October 2015.

2. Burggräf, P., Wagner, J., Koke, B. (2018), "Artificial intelligence in production management: A review of the current state of affairs and research trends in academia", in 2018 International Conference on Information Management and Processing (ICIMP), pp. 82-88.

3. Czarnota, R., Stopa, J., Janiga, D., Kosowski, P., Wojnarowski, P. (2018), "Semianalytical horizontal well length optimization under pseudosteady-state conditions", 2nd International Conference on Smart Grid and Smart Cities (ICSGSC), IEEE. 
4. Hutter, F., Hoos, H. H., Leyton-Brown, K. (2013), "An evaluation of sequential modelbasedoptimization for expensive blackbox functions", available at: https://ml.informatik.uni-freiburg.de/wp-content/uploads/papers/13-GECCOBBOB_SMAC.pdf (1 October 2021)

5. Janiga, D., Stopa, J., Mikołajczak, E., Wojnarowski, P., Czarnota, R. (2017), "Smart control of $\mathrm{CO} 2$ huff and puff process in dual porosity reservoir", International Multidisciplinary Scientific GeoConference Surveying Geology and Mining Ecology Management, SGEM, Vol. 17 No. 15, pp. 461-468.

6. Kramer, M. A. (1991), ,Nonlinear principal component analysis using autoassociative neural networks", AIChE Journal, Vol. 37 No. 2, pp. 233-243.

7. Kuk, E. (2019), "Application of Artificial Intelligence Methods to Underground Gas Storage Control", SPE Annual Technical Conference and Exhibition, Calgary, Alberta, Canada.

8. Kuk, M., Kuk, E., Janiga, D., Wojnarowski, P., Stopa, J. (2020), "Optimization wells placement policy for enhanced CO2storage capacity in mature oil reservoirs", Energies, Vol. 13 No. 16, pp. 1-20.

9. Madiraju, N. S., Sadat, S. M., Fisher, D., Karimabadi, H. (2018), "Deep temporal clustering: Fully unsupervised learning of time-domain features", available at: https://arxiv.org/pdf/1802.01059.pdf (1 October 2021)

10.Mikolajczak, E. (2016), "Computer Modeling for Intelligent Control of Gas-Condensate Reservoir", SPE Annual Technical Conference and Exhibition, SPE-184481-STU.

11.Pang, G., Shen, C., Cao, L., Hengel, A. V. D. (2020), "Deep learning for anomaly detection: a review", available at: https://arxiv.org/pdf/2007.02500.pdf (1 October 2020)

12.Ramirez, W. F. (1987), Application of Optimal Control Theory to Enhanced 816 Oil Recovery, Developments in petroleum science, Elsevier.

13.Shaowen, L., Decheng, Q., Yong, C. (2015), "Application of decision tree in xml database mining", 2015 8th International Conference on Intelligent Computation Technology and Automation (ICICTA), pp. 205-208.

14.Shteimberg, E., Kravits, M., Ellenbogen, A., Arad, M., Kadmon, Y. (2012), "Artificial intelligence in nonlinear process control based on fuzzy logic", 2012 IEEE 27th Convention of Electrical and Electronics Engineers in Israel, pp. 1-5. 


\section{About the authors}

Edyta Kuk is a Ph.D. student and a Research Assistant at the AGH University of Science and Technology. Edyta Kuk is currently with Hitachi ABB Power Grids Research, Ul. Pawia 7, 31-154 Kraków, Poland. She received her BSc in Oil and Gas Engineering in 2016, MSC in Mining and Geology in 2017, and MSC in Computer Science in 2018, all of them from AGH University of Science and Technology. Her current research interests include the oil and gas industry, mathematical modeling, artificial intelligence. She can be contacted at email kuk@agh.edu.pl

Michat Kuk is a Ph.D. student at the AGH University of Science and Technology. He received his $B S C$ in Oil and Gas Engineering from AGH University of Science and Technology in 2016 and MSC in Mining and Geology from AGH University of Science and Technology in 2017. His current research interests include gas transport, distribution networks, and the LNG market. He can be contacted at email michal.kuk@agh.edu.pl

Damian Janiga is an Adjunct Assistant Professor at AGH University of Science and Technology. He received his BSC in Oil and Gas Engineering and MSc in Mining and Geology from AGH University of Science and Technology, Ph.D. in Mining and Engineering Geology also from AGH University of Science and Technology. His current research interests include reservoir engineering, numerical simulation, optimization, and artificial intelligence. He can be contacted at email janiga@agh.edu.pl

Paweł Wojnarowski is a Professor of AGH University of Science and Technology. He received his BSC and MSC in Mining and Geology from AGH University of Science and Technology, Ph.D. in Mining and Engineering Geology also from AGH University of Science and Technology. His current research interests include reservoir engineering, numerical simulation, and EOR methods. He can be contacted at email wojnar@agh.edu.pl

Jerzy Stopa is a Professor and Chairman of the Department of Oil Engineering of the AGH University of Science and Technology. He received his BSc and MSC in Applied Mathematics from AGH University of Science and Technology in 1981 and a Ph.D. in Mining also from AGH University of Science and Technology in 1985. His current research interests include optimization, numerical simulation of oil and gas reservoirs and UGS, production forecasting. He can be contacted at email stopa@agh.edu.pl 